Journal of Case Reports 2020;10(1):48-50

\title{
Scorpion Bite Induced Myocardial Ischaemia and Pulmonary Edema
}

\author{
Ravindu Tiwari, Saumya Gupta, Abhishek Kumar, Nilesh Kumar, LP Meena \\ Department of General Medicine, Institute of Medical Sciences, Banaras Hindu University, Varanasi, Uttar Pradesh, India.
}

\begin{abstract}
Corresponding Author:
Dr. Saumya Gupta

Email: g_saumya@yahoo.com

This is an Open Access article distributed under the terms of the Creative Commons Attribution License (creativecommons.org/ licenses/by/3.0).
\end{abstract}

Received Accepted Published

October 9, 2018

February 24, 2020

March 10, 2020

\begin{abstract}
Background: Scorpion stings are common in India. Most of them are limited to moderate to severe pain at site of bite only, but good number of patients present with life threatening complications in the form of involvement of cardiac, respiratory and nervous system. Here we are reporting a case of myocarditis and pulmonary edema following sting of scorpion. Case Report: A 25 year old male presented with scorpion bite induced myocardial ischaemia, congestive cardiac failure and pulmonary edema. The cardiovascular manifestations in scorpion bite are the result of effect of venom on the sympathetic nervous system, adrenal secretion of catecholamines and the direct toxic effect of the venom on myocardium itself. Conclusion: Severe scorpion bites can present as myocardial ischaemia, congestive cardiac failure and pulmonary edema. Aggressive management with ventilator support, diuretics, parenteral steroids, antihistaminics and ionotropic agents can result in rapid clinical resolution.
\end{abstract}

Keywords: Myocarditis, Pain, Pulmonary Edema, Scorpion Sting, Venoms.

\section{Introduction}

Out of 1500 scorpion species known to exist, 30 are of medical importance. In India mainly two species are important namely, small red Buthus tamulus and large black, less dangerous but more painful Palamneus gravimanus, which causes autonomic storm, myocardial depression and pulmonary edema unlike Centruoides (found in America and Mexico) and parabuthus species (found in Africa), which causes neuromuscular toxicity. Scorpion venom is cocktail of many small molecular weight proteins which are serotonin (causing local pain), histamines and histamine releasers, neurotoxin, protease inhibitors, phospholipases, hyaluoronidases, mucopolysacchridases [1], and others of which most important are neurotoxin which causes incomplete inactivation of voltage gated sodium channels during depolarisation that result in slow inward sodium current, and due to inhibitory effect on potassium channels action potential is prolonged [2,3]. Resultant action of neurotoxins are membrane hyperexcitability, uncontrolled firing of axons which leads to release of neurotransmitters at nerve synapses and neuromuscular junctions and release of catecholamines from adrenal medulla, all causing autonomic storm [4].

\section{Case Report}

A 25 year old male presented to us with chief complain of breathlessness, fever and three episodes of vomiting for 6 hours, after sting of scorpion on toes. There was no history of any comorbidity. On examination his general condition was poor, he was tachypneic with respiratory rate of $40 / \mathrm{min}$, pulse of 120 beats per minute, blood pressure $80 / 60 \mathrm{~mm} \mathrm{Hg}$, peripheral extremities were cold and clammy. On systemic examination sensorium was intact with GCS of 15, S3 gallop was present on cardiovascular examination and bibasilar fine crepitations were present on both sides of thorax on auscultation. 
His arterial blood gas analysis showed arterial oxygen saturation $85 \%, \mathrm{PaO}_{2} 65.7 \mathrm{~mm} \mathrm{Hg}$, $\mathrm{pH} 7.3, \mathrm{HCO}_{3} 26.6 \mathrm{mmol} / \mathrm{L}$, anion gap $18.3 / \mathrm{L}, \mathrm{PaO}_{2}$ (partial pressure of arterial oxygen tension) $/ \mathrm{FiO}_{2}$ $(\mathrm{P} / \mathrm{F})$ ratio 122.4 . His serum cardiac biomarker test shown CKMB $7.54 \mathrm{ng} / \mathrm{mL}$ (1.39-6.32), troponin I $0.6 \mathrm{ng} / \mathrm{mL}$ (0.000-0.014), BNP $2520 \mathrm{pg} / \mathrm{mL}$, blood sugar was $130 \mathrm{mg} / \mathrm{dL}$. His chest $\mathrm{x}$-ray showed parahillar infiltrations and cephalisation suggesting pulmonary edema [Fig.1]. His ECG showed sinus tachycardia, ventricular premature beats, ST segment elevations in leads II,III, aVF [Fig.2]. Echocardiogram demonstrated hypokinesia of the inter-ventricular septum and postero-inferior wall with ejection fraction of $36 \%$. A diagnosis of myocardial ischaemia with pulmonary edema due to scorpion sting was made. Patient was intubated and nasogastric tube was inserted. Tablet prazosin $1 \mathrm{mg}$ ever $6 \mathrm{hrly}$, aspirin and nitrates were given through nasogastric tube. Diphenhydramine and corticosteroid injection were given in view of severe anaphylaxis reaction. Dobutamine infusion was started, diuretics were given for shock and congestion. After 7 days of ICU stay he was shifted to general ward and discharged after next 2 days with stable vitals.

\section{Discussion}

The scorpion venom is a water soluble antigenic complex mixture of neurotoxins, cardiotoxins, nephrotoxin, hemolysin, histamine and other chemicals. The primary target of scorpion venom is voltage gated ion channels. The venom produces both local and systemic reactions. Local reaction consists of itching, edema, ecchymosis and burning pain. Clinical effects of scorpion envenomation depend on the species of scorpion, lethality and dose of venom injected. Autonomic storm evoked due to envenomation is characterized by transient parasympathetic (vomiting, profuse sweating, cold extremities, hypotension, bradycardia, and in some cases priapism) activity. Prolonged massive release of catecholamines later produces restlessness, tachycardia, hypertension, cardiac failure and pulmonary edema.

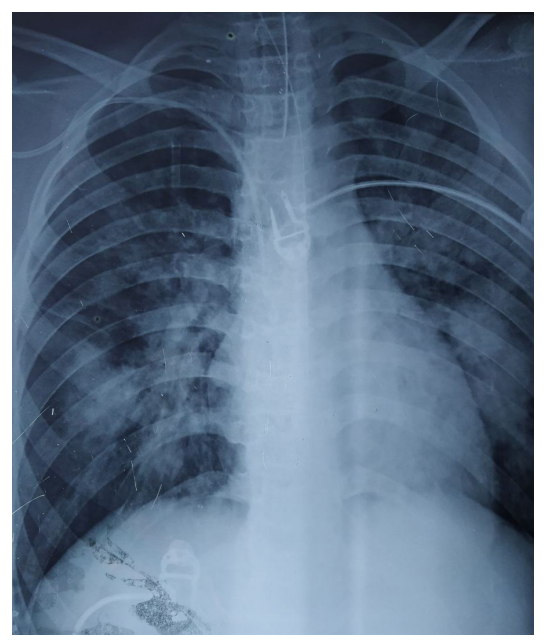

Fig.1: Chest $x$-ray suggestive of pulmonary edema.
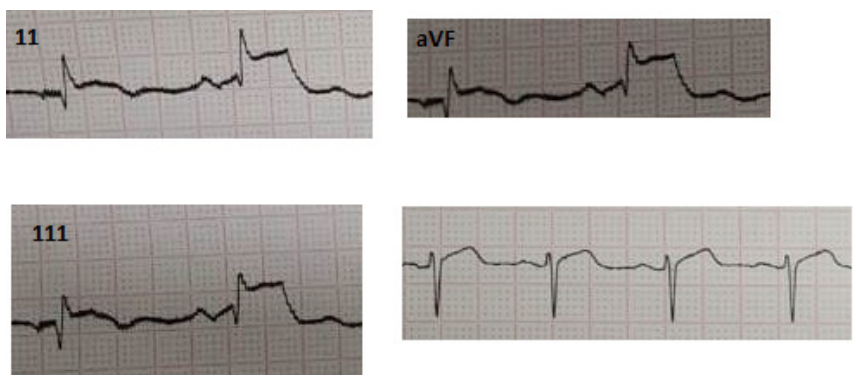

Fig.2: ECG showed sinus tachycardia, ventricular premature beats, ST segment elevations in leads II,III, aVF.

Cardiac involvement is due to both myocardial ischemia by coronary spasm (due to vasoactive, inflammatory and thromboenic peptides and amino constituents like serotonin, histamines, leukotriens and thromboxane) [5], and myocardium toxicity (due to overstimulation by catecholamines due to autonomic storm and direct effect of venom) [6]. Pulmonary edema develops due to myocardial hypofunctioning and also due to bradykinins which gets accumulated as their degradation is prevented by scorpion toxin [7]. ECG abnormalities in the form of ST segment and T wave changes are common in severe envenomation. Early myocardial infarction like pattern atrial arrhythmias, ventricular premature beats, tachycardia, QT prolongation hemiblock low voltage and wide QRS complex has been demonstrated in scorpion venom myocarditis in different combinations [8,9]. Prazosin is 
pharmacological and molecular antidote [10] and can be given when there is hypotension, because of which it is called poor man's scorpion antivenom [11]. Hypotension can be managed with dobutamine [12]. Pulmonary edema should be managed with judicial use of diuretics to prevent dehydration, mechanical ventilation and nitroglycerine infusion as later has venodilatory action and reduces preload [13]. Early treatment is of paramount importance for favourable prognosis. Scorpion antivenom should be used according to availability, as it enhances recover time and reduces hospital stay [14].

\section{Conclusion}

Scorpion bites can produce life threatening cardiac complications and can affect any age group. Early recognition and aggressive management with antiischaemic therapy and adequate cardiorespiratory support can result in favourable clinical outcomes. A routine electrocardiogram should be done to look for cardiac complications and if required can be accompanied with serum cardiac biomarkers and echocardiogram.

Contributors: RT and SG conceived the presented idea, as well as drafted and revised the manuscript in consultation with AK, NK and LPM. SG will act as a study guarantor. All authors have read and approved the final manuscript and are accountable for all aspects of the final manuscript.

Funding: None; Competing interests: None stated.

\section{References}

1. Bawaskar HS, Bawaskar PH. Scorpion sting. J Assoc Physicians India. 1998;46:388-392.

2. Possani LD, Baltazar B, Delepierre M, Tygat J. Scorpion toxins specific for NA+ channels. Eur J Biochem. 1999;264:287-300.
3. Gwee MCE, Nirtthanan S, Khoo H, Gopalkrishnakone P, Kini MR, Cheah LS. Autonomic effects of same scorpion venoms and toxins. Clin Exp Pharmacol Physiol. 2002;29:795-801.

4. Bawaskar HS, Bawaskar PH. Sting by red scorpion (Buthotus Tamulus) in Maharashtra state, India: A clinical study. Trans Roy Soc Trop Med Hyg. 1989;83:858-860.

5. Yang HP, Chen FC, Chen CC, Shen TY, Wu SP, Tseng YZ. Manifestations mimicking acute myocardial infarction after Honeybee Sting. Acta Cardiol Sin. 2009;25:31-35.

6. Rahav G, Weiss T. Scorpion sting induced pulmonary edema. Scintigraphic evidence of cardiac dysfunction. Chest. 1990;97:1478-1480.

7. Bawaskar HS, Bawaskar PH. Management of scorpion sting. Heart. 1999;82:253-2524.

8. Amaral CFS, Lopes JA, Magalhaes RA, Rezende NA. Electrocardigraphic enzymatic and echocardiographic evidence of myocardial damge after Tityus Serrulatus scorpion poisoning. Am J Cardiol. 1991;67:655-657.

9. Poon-king T. Myocarditis from scorpion sting. Br Med J. 19631:374-376.

10. Gupta V. Prazosin: A Pharmacological antidote for scorpion envenomation. J Trop Peditr. 2006;52:150-151.

11. Bawaskar HS, Bawaskar PH. Prazosin in management of cardiovascular manifestations of scorpion sting. Lancet. 1986;2:510-511.

12. Eltrous S, Nouria S, Besbes-Ouanes L, Boussarsar M, BoukefR, Marghli S, et al. Dobutamine in severe scorpion envenomation effects on standard hemodynamics, right ventricular performance, and tissue oxygenation. Chest. 1999;116:748-753.

13. Narayanan P, Mahadevan S, Serane VT. Nitroglycerine in scorpion sting with decompensated shock. Indian Pediatr. 2006;43:613-617.

14. Bawaskar HS, Bawaskar PH. Utility of scorpion antivenom Vs prazosin in the management of severe Mesobuthus tamulus envenoming at rural setting. J Assoc Physicians India. 2007;55:14-21. 